\title{
On the Use of Experimental Methods to Improve Confidence in Educed Impedance
}

\author{
M. G. Jones*and W. R. Watson ${ }^{\dagger}$
}

NASA Langley Research Center, Hampton, VA 23681

\begin{abstract}
Results from impedance eduction methods developed by NASA Langley Research Center are used throughout the acoustic liner community. In spite of recent enhancements, occasional anomalies persist with these methods, generally at frequencies where the liner produces minimal attenuation. This investigation demonstrates an experimental approach to educe impedance with increased confidence over a desired frequency range, by combining results from successive tests with different cavity depths. A series of tests is conducted with three wire-mesh facesheets, for which the results should be weakly dependent on source sound pressure level and mean grazing flow speed. First, a raylometer is used to measure the DC flow resistance of each facesheet. These facesheets are then mounted onto a frame and a normal incidence tube is used to determine their respective acoustic impedance spectra. A comparison of the acoustic resistance component with the DC flow resistance for each facesheet is used to validate the measurement process. Next, each facesheet is successively mounted onto three frames with different cavity depths, and a grazing flow impedance tube is used to educe their respective acoustic impedance spectra with and without mean flow. The no-flow results are compared with those measured in the normal incidence tube to validate the impedance eduction method. Since the anti-resonance frequency varies with cavity depth, each sample provides robust results over a different frequency range. Hence, a combination of results can be used to determine the facesheet acoustic resistance. When combined with the acoustic reactance, observed to be weakly dependent on the source sound pressure level and grazing flow Mach number, the acoustic impedance can be educed with increased confidence. Representative results of these tests are discussed, and the complete database is available in electronic format upon request.
\end{abstract}

\section{Nomenclature}

$\begin{array}{ll}c_{0}, \rho_{0} & =\text { sound speed and density of air, respectively, } \mathrm{m} / \mathrm{s} \text { and } \mathrm{kg} / \mathrm{m}^{3} \\ d & =\text { cavity depth, } m \\ i & =\sqrt{-1}, \text { unit imaginary number } \\ F(\zeta) & =\text { wall error function, } P a^{2} \\ k & =\omega / c_{0}, \text { freespace wavenumber, } \mathrm{m}^{-1} \\ L_{1}, L_{2} & =\text { axial locations of liner leading and trailing edges, respectively, } m \\ L & =\text { axial location of exit plane for computational domain, } \mathrm{m} \\ M_{0} & =U_{0} / c_{0}, \text { uniform mean flow Mach number } \\ N L F & =\text { nonlinearity factor } \\ p & =\text { acoustic pressure, } P a \\ R_{f} & =\text { DC flow resistance, } M K S \text { Rayls }\end{array}$

* Senior Research Scientist, Research Directorate, Structural Acoustics Branch, AIAA Associate Fellow.

†Senior Research Scientist, Research Directorate, Computational AeroSciences Branch, AIAA Associate Fellow. 


$$
\begin{array}{ll}
\zeta, \theta, \chi & =\text { normalized acoustic impedance, resistance and reactance, respectively, } \zeta=\theta+i \chi \\
U_{0} & =\text { uniform axial flow velocity, } m / s \\
\omega & =\text { angular frequency, } s^{-1} \\
x, y, z & =\text { axial, vertical and spanwise dimensions of flow duct, respectively, } m \\
x_{I} & =\text { axial location of microphone, } m \\
\zeta_{u} & =\text { normalized acoustic impedance of liner on upper wall }
\end{array}
$$

Note: An $e^{i \omega t}$ time convention is used throughout this paper, and all impedances are normalized by $\rho_{0} c_{0}$, the characteristic impedance of air.

\section{Introduction}

There is constant pressure to reduce aircraft noise in the vicinity of airports, even as the volume of air traffic continuously increases. This desire for reduced aircraft noise has led the Federal Aviation Administration and International Civil Aviation Organization to impose increasingly stringent noise requirements, and has resulted in additional noise constraints for selected airports. In response to this concern, NASA's Fundamental Aeronautics Program has set a goal to confine objectionable aircraft noise within the airport boundaries via innovative noise reduction concepts. This goal is well beyond the current capabilities of the commercial aircraft industry, and will require revolutionary changes.

One of the dominant noise sources for subsonic aircraft is fan noise produced by the engine. Over the last four decades, significant effort has been directed toward the reduction of this noise source via modifications to the fan geometry, e.g., lean and sweep $^{1}$ of the fan blades. During the last decade, additional effort has been directed toward novel concepts to further reduce aircraft fan noise. Novel concepts currently being investigated by NASA include soft vanes and over-the-rotor liners. ${ }^{2,3}$ However, the best method for fan noise reduction is the use of acoustic liners mounted in the aircraft engine nacelles. Although liners already provide a significant portion of the current fan-noise reduction, the challenging goal to confine aircraft objectionable noise within aircraft boundaries highlights the need to further optimize these liners to maximize noise reduction.

The key parameter used to understand noise reduction achieved with an acoustic liner is acoustic impedance, an intrinsic parameter that is dependent on sound pressure level and grazing flow velocity. A number of methods have been developed to determine acoustic impedance in the presence of grazing flow. In 1969, Feder et al ${ }^{4}$ presented a "T-Tube" approach to measure the acoustic impedance drop across a perforated facesheet, in which one side of the sheet is exposed to sound while the other side is exposed to grazing flow. More recently, Syed et $a l^{5}$ used a related approach, in which the DC flow resistance of the perforated facesheet is measured in the presence of grazing flow. In this approach, the frequency-dependent, acoustic resistance of the facesheet is assumed equal to the DC flow resistance, an assumption that holds reasonably well for frequencies away from the resonance and anti-resonance frequencies of the liner. Neither method provides information regarding the contribution of the liner cavity (generally filled with honeycomb), but they instead rely on impedance prediction models for that component. This is a reasonable approach, as the acoustic reactance is weakly dependent on the grazing flow velocity. Although these methods require additional instrumentation (side-branch tube, etc.), they enable eduction of the acoustic impedance of the liner in the presence of grazing flow without the need for a duct propagation code.

In 1974, Dean ${ }^{6}$ presented a two-microphone in situ method for the direct measurement of acoustic impedance for single-layer liners. Malmary et al ${ }^{7}$ replaced one of the two microphones with a probe microphone to measure acoustic pressure at multiple penetration depths within the liner, and Zandbergen ${ }^{8}$ extended Dean's approach to use three embedded microphones with two-layer liners. These in situ methods provide a direct measurement of the acoustic impedance, but have the disadvantage that they require embedding of microphones into the liner and provide only localized information in the vicinity of these microphones. Nevertheless, this general approach is used by multiple companies because it provides a direct measurement of the liner impedance. Another method has been implemented more recently to allow direct measurement of the sound field in the vicinity of the liner surface, again with the liner mounted in the wall of a flow duct. This method uses Laser Doppler Velocimetry to measure the acoustic velocity field above the 
liner surface, which is then used to educe the liner impedance..$^{9,10}$

A third category of impedance eduction methods is based on the combination of a duct propagation code and measurements in the hardwall sections upstream and/or downstream of a liner mounted in one wall of a flow duct. One such method is the mode-matching method, ${ }^{11-13}$ in which complex acoustic pressures are measured in the hardwall sections upstream and downstream of the liner, and are then used to determine the modal content in these hardwall sections. This modal information is combined with a multi-modal duct propagation code to determine the liner impedance. For those conditions where only plane waves propagate in the hardwall sections, conventional two-microphone methods ${ }^{14,15}$ can be used to determine the acoustic field upstream and downstream of the liner. ${ }^{16,17}$ Multiple researchers ${ }^{5,18,19}$ have also combined propagation codes with insertion loss measurements to educe the liner impedance. Essentially, the impedance of the liner is iterated upon in the duct propagation code until the measured and predicted insertion losses are matched. However, since a particular insertion loss can be achieved via multiple impedances, additional information is required to determine the liner impedance. Whereas the aforementioned methods use frequency-domain analysis, Fung $e t a l^{20}$ generated pulses upstream of the lined section of the flow duct, and used the resultant reflections to educe the liner impedance in a time-domain approach.

The last impedance eduction category involves the measurement of acoustic pressures on the wall opposite of the acoustic liner, again mounted in one wall of a flow duct. In 1974, Armstrong $e t$ al ${ }^{21}$ presented a method that uses this acoustic pressure profile to determine the axial wavenumber of the least attenuated mode, from which the acoustic impedance of the liner can be directly inferred. Succi ${ }^{22}$ used a similar approach to educe the impedance for both local and extended-reaction liners. In 1987, Parrott et al ${ }^{23}$ used a finite element model with this acoustic pressure data to compare the effects of mean flow profile on the sensitivity of the impedance eduction process. Eversman et $a l^{24}$ used a similar method that includes the effective uniform-flow Mach number in the eduction process, i.e., the impedance and Mach number are both assumed unknown, and are jointly iterated upon within the finite element duct propagation code until they produce the measured acoustic pressure profile.

Over the last three decades, the NASA Langley Liner Physics Team has investigated a number of these impedance eduction methods, with emphasis on the continued development of (1) duct propagation codes with increased fidelity, (2) optimization schemes to allow efficient determination of the liner impedance, and (3) test rigs suitable for evaluation of acoustic liners in realistic aeroacoustic environments. These impedance eduction methods are based on three sets of equations; the convected Helmholtz equation, which only supports uniform flow, and the linearized Euler equations and the Pridmore-Brown equation, which include shear-flow effects. ${ }^{25,26}$ The methods based on the convected Helmholtz (CHE) and linearized Euler (LEE) equations assume a finite-length acoustic liner mounted in a rectangular waveguide, while the method based on the Pridmore-Brown (PBE) equation assumes an infinite-length acoustic liner mounted in a rectangular waveguide. Briefly, the finite-length liner impedance eduction methods (CHE and LEE) are based on iterating the liner impedance until the acoustic pressures predicted by a duct propagation code match the acoustic pressures measured at a number of microphone locations to within an acceptable tolerance. For those cases where a single mode is dominant over a significant portion of the liner, the infinite-length liner impedance eduction method (PBE) uses amplitude and phase decay rates over the axial extent of the liner to directly compute the liner impedance.

Previous studies based on these three impedance eduction methods have, in general, provided excellent results that continue to be used throughout the acoustic liner community. However, these studies have also demonstrated a number of isolated cases in which the results were considered to be anomalous; i.e., the educed impedance did not follow expected trends. These anomalous results have been observed with each of these methods. In 2001, Jones et $a l^{27}$ compared results achieved with the CHE and PBE (labeled as SMM in that paper) methods. These methods were shown to provide similar results, and the authors suggested use of the PBE method whenever possible due to its ease of usage. However, both methods exhibited anomalous behavior at frequencies near anti-resonance. Similar anomalous results have been observed by Watson et al for the CHE and LEE methods, ${ }^{28-30}$ for frequencies near anti-resonance or the cut-on frequency for higher-order modes.

In response to these results, a series of modifications to these impedance eduction methods was initiated. The first of these was an enhancement to the CHE and LEE methods, in which the exit impedance boundary condition was replaced with an exit pressure boundary condition. Due to the manner in which data are acquired in the NASA Grazing Flow Impedance Tube (GFIT), the exit pressure condition can 
be directly acquired, whereas the exit impedance condition involves the use of a model that (1) requires that only plane waves are present, (2) assumes the termination to be nearly anechoic, ${ }^{30}$ and (3) requires complex acoustic pressure measurements at two or more axial locations. This enhancement reduced the occurrence of anomalous results, but did not eliminate them. In 2010, Watson ${ }^{31}$ incorporated three additional enhancements to the CHE impedance eduction method. First, a new sparse equation solver was implemented that allows for iterative refinement, a process to reduce the effects of numerical round-off errors in the equation solver. Second, constraints were incorporated into the optimization algorithm, such that only positive resistances are allowed for passive liners. The earlier version of the duct propagation code had included all impedances in the evaluation space, whether or not they were physically realizable, resulting in occasional educed impedances with negative resistances (i.e., suggesting an acoustic source embedded within the liner). Finally, multiple (typically, four) distinct starting locations were implemented to increase the likelihood of finding a global optimum. These enhancements significantly improved the results, but still did not completely eliminate anomalous behavior. Detailed attenuation contour maps as a function of liner impedance were used to demonstrate that many of these anomalies occurred at frequencies for which the objective function was extremely flat, i.e., at frequencies for which sizable changes in the liner impedance result in minimal changes in the attenuation due to the liner.

It has long been understood that there is an optimum impedance for a given waveguide geometry. In 1953. Cremer ${ }^{32}$ used a modal analysis of sound propagation through a rectangular duct to determine the liner impedance that would produce maximum attenuation in the absence of mean flow. Tester ${ }^{33}$ later extended the model to predict the impedance that produces maximum attenuation when grazing flow effects are included. Tester also showed the attenuation to be a strong function of impedance in the vicinity of this optimum, i.e., slight changes in liner impedance result in significant changes in attenuation. This causes the uncertainty in the predicted attenuation to increase near this optimum frequency, making it challenging to predict attenuation accurately in this frequency regime. On the other hand, the uncertainty in the educed impedance is significantly reduced in this frequency regime. Thus, it appears obvious that the strong dependence of sound attenuation on the liner impedance should increase the robustness of impedance eduction processes for impedances near this optimum.

This study seeks to exploit this fact to demonstrate a methodology for which the liner impedance can be educed with increased confidence across the entire frequency range of interest. First, the test liner is mounted in the GFIT and an impedance eduction process is used to estimate the liner impedance. Next, the facesheet is remounted onto a frame with a different cavity depth, and the impedance eduction process is repeated. In an ideal situation with no anomalous results, the main difference between the two impedance spectra will be in the cavity reactance, given by $-\cot (k d)$. For those cases where anomalous results occur, it is expected that at least one of the cavity depths (three cavity depths are used in the current study) will bring the liner impedance sufficiently close to the optimum impedance to increase the attenuation and thereby increase the robustness of the impedance eduction process. Thus, the combination of results achieved with multiple cavity depths can be used to increase confidence in the impedance eduction process over a wide frequency range.

The purpose of the current study is to further investigate the effects of these flat objective functions on the NASA Langley impedance eduction process, and to explore the hypothesis that the resistance of the liner facesheet can be confidently educed when the cavity depth is chosen to maximize attenuation. This study is conducted with test samples purposefully selected for their linear properties, such that the effects of acoustic resistance and reactance coupling is minimal. Three types of wire mesh facesheets are combined with three honeycomb-filled holders, each with a cavity depth selected to optimize attenuation over a different frequency range, creating nine mesh-over-honeycomb liners. Component tests are conducted with a raylometer and a normal incidence impedance tube to measure the DC flow resistance and no-flow acoustic impedance, respectively, of each liner configuration. These component results are then used to validate impedances educed for each of these liner configurations based on acoustic pressures measured in the GFIT, with and without grazing flow. A description of the aeroacoustic propagation model is provided in Section II. Section III provides a description of the test samples and experimental methods used in this investigation. Results are provided in Section IV, and concluding remarks regarding some of the more signicant results are summarized in Section V. 


\section{Aeroacoustic Propagation Model}

It was mentioned earlier that, although the impedance eduction methods used by NASA generally provide excellent results, they are prone to occasional anomalous results, especially for liner configurations that produce minimal attenuation in the GFIT. For convenience, the current investigation focuses on the impedance eduction method based on the convected Helmholtz equation (CHE), with the expectation that results achieved with this method will also provide insight regarding the other methods.

The analysis used in the current investigation has been presented in a previous paper, ${ }^{34}$ and is summarized here for convenience. Figure 1 provides a schematic of the three-dimensional flow duct and Cartesian coordinate system used in the aeroacoustic propagation model. The surface of the liner with unknown, uniform, normal-incidence, normalized impedance, $\zeta$, comprises a portion of the upper wall of the flow duct over the axial extent of $L_{1} \leq x \leq L_{2}$. Upstream and downstream of the liner, the flow duct upper wall is rigid. The lower and two side walls are also rigid, and the source and exit planes of the computational domain are located at $x=0$ and $x=L$, respectively. The sound source is assumed to be a single tone at a frequency such that no higher-order horizontal modes propagate, thereby allowing the analysis to be restricted to the $(x, y)$-plane.

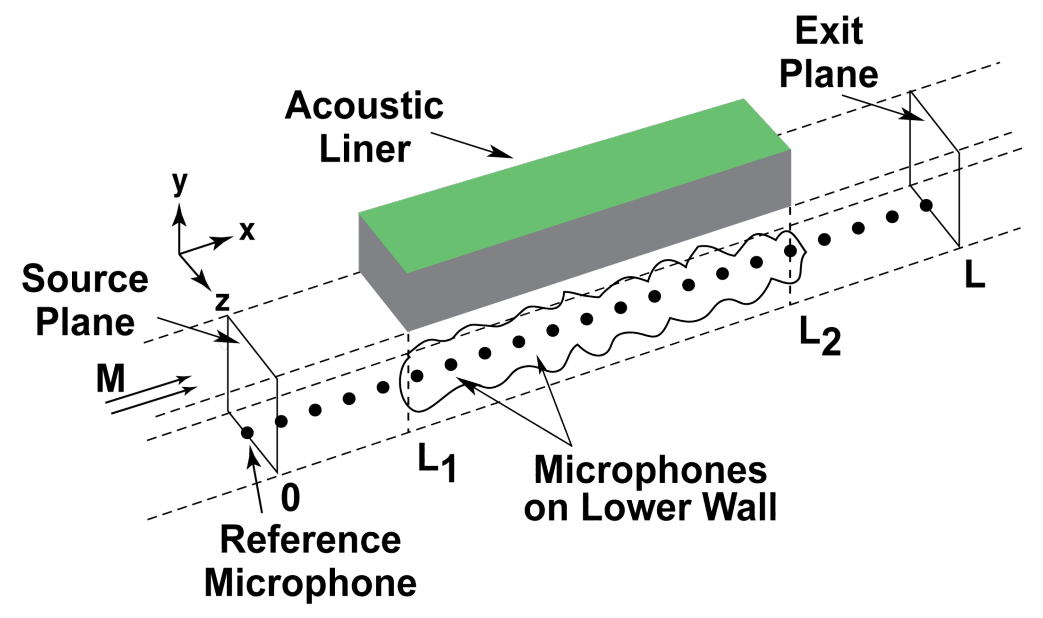

Figure 1: Sketch of Grazing Flow Impedance Tube computational domain.

Throughout the flow duct, the acoustic pressure field, $p$, is assumed to satisfy the convected Helmholtz equation

$$
\left(1-M_{0}^{2}\right) \frac{\partial^{2} p}{\partial x^{2}}+\frac{\partial^{2} p}{\partial y^{2}}-2 i k M_{0} \frac{\partial p}{\partial x}+k^{2} p=0
$$

Mean flow profiles measured just upstream and downstream of the computational domain are used to compute average axial flow velocities, and a linear fit between the two axial locations is used to determine the uniformflow axial velocity, $U_{0}$. The liner is assumed to be locally-reacting, and to be properly represented by the Myers $^{35}$ wall impedance boundary condition, given by

$$
-\frac{\partial p}{\partial y}=i k\left(\frac{p}{\zeta_{u}}\right)+2 M_{0} \frac{\partial}{\partial x}\left(\frac{p}{\zeta_{u}}\right)+\frac{M_{0}^{2}}{i k} \frac{\partial^{2}}{\partial x^{2}}\left(\frac{p}{\zeta_{u}}\right)
$$

where $\zeta_{u}$ represents the impedance of the upper wall. The admittance (i.e., $1 / \zeta_{u}$ ) is taken as zero for rigid upper wall sections of the duct located upstream and downstream of the liner, and $\zeta_{u}=\zeta$ is used in the liner portion of duct. The boundary condition of the rigid lower wall is

$$
\frac{\partial p}{\partial y}=0
$$


Recall that the sound source is assumed to be a single tone at a frequency such that no higher-order horizontal modes propagate. For this condition, the acoustic pressure profile at the source plane, $p(0, y)$, is assumed constant, and is taken to be equal to the acoustic pressure measured at the reference microphone on the lower wall, $p(0,0)$. This can be expressed as the source plane boundary condition

$$
p(0, y)=p(0,0)
$$

Similarly, the exit plane acoustic pressure boundary condition is based on the acoustic pressure measured by the microphone on the lower wall at $x=L$, and is expressed as

$$
p(L, y)=p(L, 0)
$$

Note that the acoustic pressure measurements must be obtained with the test liner installed. Equations (1)-(5) do not have exact solutions for a general set of input parameters. Consequently, they are solved numerically using a finite element method. An essential feature of the impedance eduction technique employed in this paper is the development of a general purpose numerical procedure to determine the impedance that reproduces the acoustic pressure field measured on the wall opposite the test liner. The wall error function

$$
F(\zeta)=\sum_{I=1}^{n w a l l}\left\{\left.p\left(x_{I}, 0\right)\right|_{\mathrm{FEM}}-\left.p\left(x_{I}, 0\right)\right|_{\text {Meas }}\right\}\left\{\left.p^{*}\left(x_{I}, 0\right)\right|_{\mathrm{FEM}}-\left.p^{*}\left(x_{I}, 0\right)\right|_{\text {Meas }}\right\}
$$

is used as an objective function, and the unknown impedance, $\zeta$, is selected such that this objective function is minimized. The superscript ${ }^{*}$ represents the complex conjugate of the corresponding parameter. The above objective function may be interpreted as the difference between the measured, $\left.p\left(x_{I}, 0\right)\right|_{\text {Meas }}$, and numerically computed, $\left.p\left(x_{I}, 0\right)\right|_{\mathrm{FEM}}$, wall pressures opposite the test liner at nwall microphone locations. Note that the proposed procedure is perfectly general and requires only that the formulation of the duct propagation model constitute a well-posed boundary value problem.

An initial guess of the liner impedance is required for the eduction process. For the current investigation, four independent optimizations are conducted for each test condition, each with a unique starting value of the liner impedance. For this study, these starting values were $0.5+2 i, 0.5-2 i, 2+2 i$, and $2-2 i$. A comparison of the four resultant educed impedances is used to determine whether any of the results were due to local optimima. If all four values are identical, the result is generally assumed to be a global optimum. If any of the values differs from the remaining values, physical reasoning is applied to determine which one is anomalous. As mentioned earlier, the resistance is generally assumed to be frequency-independent at frequencies away from resonance and anti-resonance, and the reactance is assumed to be dominated by a $-\cot (k d)$ pattern. It should be noted that the current study was conducted with an unconstrained optimization methodology, such that non-physical acoustic resistances can be educed. To correct for this issue, an additional criterion is applied to eliminate anomalies, i.e., the resistance must be positive for a passive liner.

\section{Experimental Methods}

Successive tests in the NASA Langley raylometer, normal incidence tube (NIT) and grazing flow impedance tube (GFIT) are used for detailed evaluation of each liner configuration. Raylometer results are used to validate the NIT results, and NIT results are used to validate GFIT results (at no flow). First, wire mesh facesheets are tested in the raylometer to determine their DC flow resistance, assumed equivalent to the acoustic resistance at $0 \mathrm{~Hz}$, and their nonlinearity factor, $N L F$. Since wire mesh is expected to have a low $N L F$, the DC flow resistance should represent a good approximation for the acoustic resistance at frequencies away from resonance and anti-resonance. These facesheets are then mounted onto a frame for testing in the NIT, such that the validity of this assumption can be demonstrated. These NIT tests provide the acoustic impedance spectra for each liner, comprised of the acoustic resistance, predominately a function of the facesheet resistance, and the acoustic reactance, a function of the cavity depth and the mass reactance of the wire mesh facesheet. Next, larger versions of the same samples are tested in the GFIT, such that the effects of grazing incidence sound and mean flow can be determined. Initial GFIT tests are conducted in the absence of mean flow, such that the normal incidence acoustic impedance should match that measured in the 


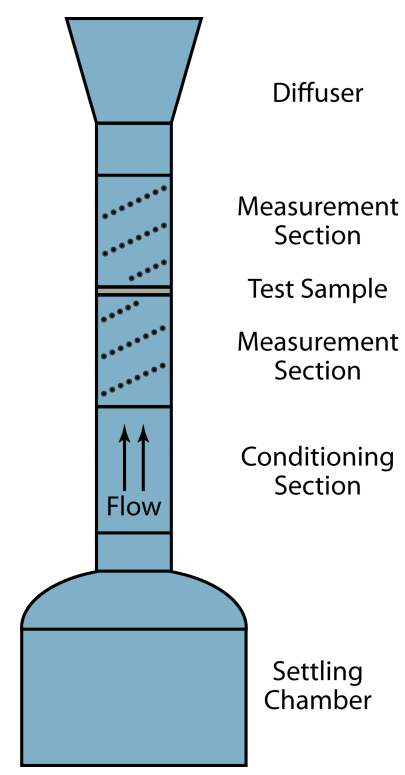

(a) Raylometer.

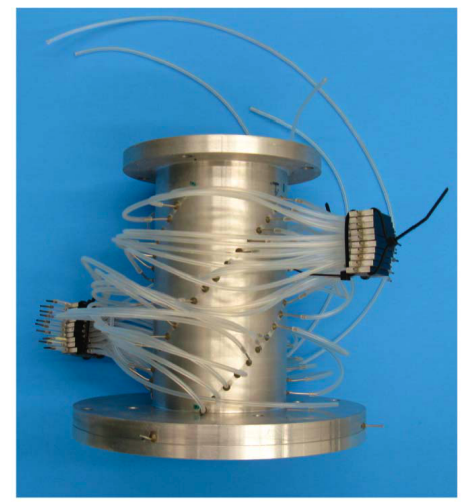

(b) Measurement section.

Figure 2: Sketch of NASA Langley raylometer with detailed view of measurement section with spiral arrays.

NIT. Finally, GFIT tests are conducted in the presence of mean flow, such that the effects of grazing flow can be assessed. (As a side note, it should be noted that although the impedance educed in this environment is commonly labeled as grazing flow impedance, it is based on the normal-incident component of acoustic particle velocity and is therefore the normal incidence acoustic impedance of the liner.)

\section{A. Raylometer}

Three wire mesh facesheets are used in the current study, designed by the manufacturer to provide DC flow resistances, $R_{f}$, of 150,270 and 570 MKS Rayls $\left(\mathrm{kg} \cdot \mathrm{m}^{-2} \cdot \mathrm{s}^{-1}\right)$. For convenience, these materials are labeled as R1, R2 and R3, respectively. A $50.8 \mathrm{~mm} \times 50.8 \mathrm{~mm}$ sample of each type was tested in the NASA raylometer. Care was taken in the mounting process to hold the test material tight, thereby minimizing vibration (flapping) and forcing all of the air to pass through the material, i.e., to avoid a shunt path that would cause the measured flow resistance to be arbitrarily low.

A sketch of the NASA raylometer is provided in Fig. 2. Mass flowmeters are controlled to provide metered air into the settling chamber. The air flows up from the settling chamber, through a conditioning section that transitions from a round to square inner geometry, and into the lower measurement section. The outer surface of the measurement section is circular (see Fig. 2-b), but the inner surface is square, such that the measurement section properly mates with the square sample. Figure 2-b provides a detailed view of this section with 80 static pressure taps, divided into four arrays positioned circumferentially around the duct in $90^{\circ}$ increments. Each set of static pressure ports is arranged in a spiral array around the circumference of the flow tube to minimize interference between axially adjacent measurements.

The air flow continues through the sample into a second, identical measurement section and out through the diffuser section. Air flow velocities of 1 to $5000 \mathrm{~mm} / \mathrm{s}$ can be generated with this device. Tests are conducted at a series of target flow velocities. At each test velocity, the static pressures are measured upstream and downstream of the test material. Curve-fits to the pressure profiles acquired with each spiral array are used to compute the static pressures on each side of the test material, such that the static pressure drop, $\Delta p_{s}$, across the test material can be determined with confidence. The DC flow resistance at a selected through-flow velocity, $u$, is computed as $R_{f}(u)=\Delta p_{s} / u$. 


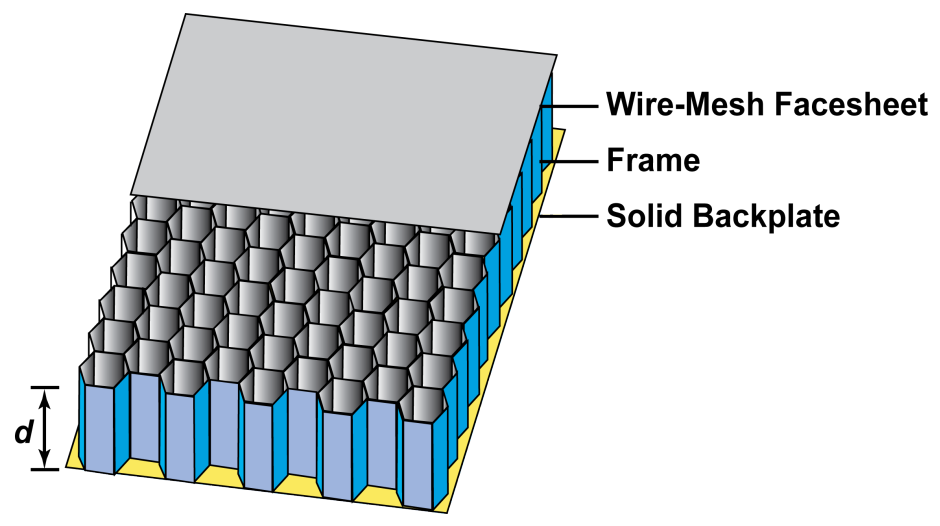

Figure 3: Sketch of sample used with NIT.

\section{B. Normal Incidence Tube}

Four samples were tested in the NASA normal incidence tube (NIT). The first three samples (see Fig. 3) are those tested in the raylometer, i.e., three wire mesh facesheets successively mounted onto a $50.8 \mathrm{~mm}$-deep frame, while the last sample uses the same frame with no facesheet. A sketch of the NASA NIT is provided in Fig. 4. It contains six $120-\mathrm{W}$ compression drivers to generate a plane-wave sound field that impinges on the surface of the sample and combines with reflections from the sample to create a standing wave pattern. The Two-Microphone Method ${ }^{14,15}$ is used to measure the complex acoustic pressures at two prescribed distances from the liner surface, such that the frequency dependence of the no-flow acoustic impedance of the liner can be computed. These tests are conducted with discrete tones, one frequency at a time. Tests are conducted for source frequencies of 0.4 to $3.0 \mathrm{kHz}$ in $0.2 \mathrm{kHz}$ increments, with source sound pressure levels (SPLs) of 110 and $130 \mathrm{~dB}$ at the reference microphone, respectively. Two source amplitudes are used, such that test sample nonlinearities can be evaluated by determining whether the impedance is a function of the reference SPL.

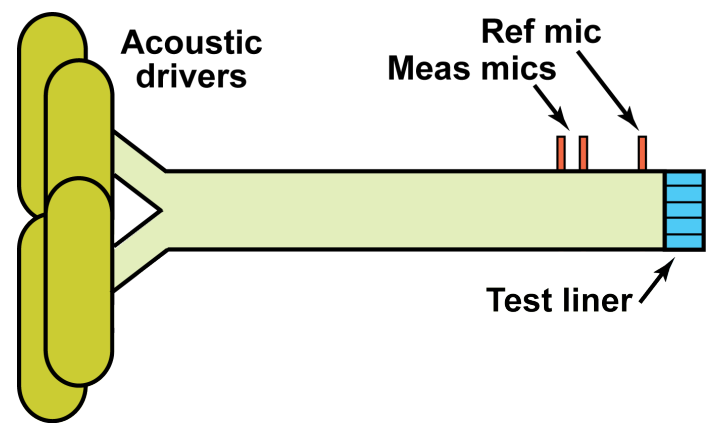

Figure 4: Sketch of NASA normal incidence tube (NIT).

\section{Grazing Flow Impedance Tube}

Nine liner configurations (see Fig. 5) are considered for this portion of the study. These liners are implemented via a modular approach, in which three frames with depths of 38.1, 50.8 and $76.2 \mathrm{~mm}$, respectively, are combined with three wire mesh facesheets (R1, R2 and R3) to construct nine liner configurations. The frames are constructed with aluminum, with wall thicknesses of $6.35 \mathrm{~mm}$, outer dimensions of $63.5 \mathrm{~mm} \times 609.6 \mathrm{~mm}$, and inner dimensions of $50.8 \mathrm{~mm} \times 596.9 \mathrm{~mm}$. 


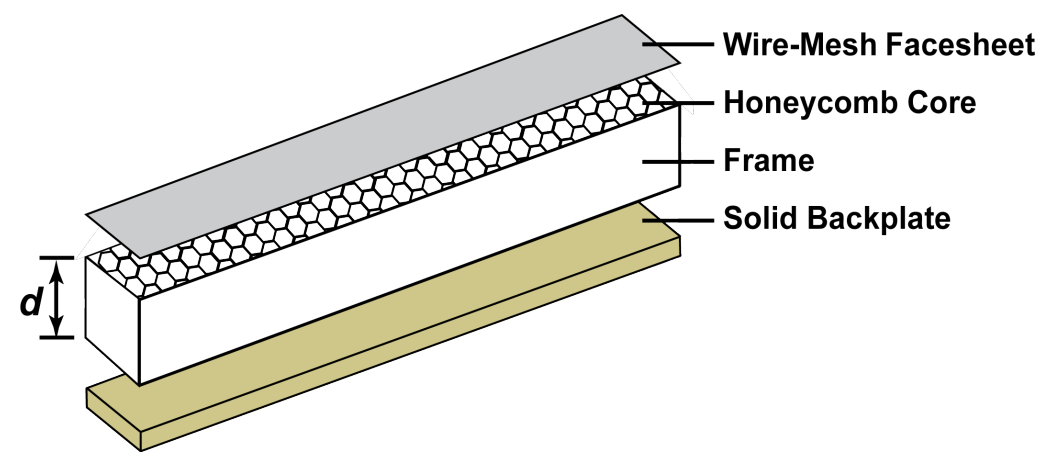

Figure 5: Sketch of sample used with GFIT.

These liners are tested in the NASA grazing flow impedance tube (GFIT). This waveguide has a crosssectional geometry of $50.8 \mathrm{~mm} \times 63.5 \mathrm{~mm}$, such that higher-order modes in the horizontal and vertical dimensions cut on at different frequencies. It allows convenient evaluation of acoustic liners with lengths from $50.8 \mathrm{~mm}$ to $609.6 \mathrm{~mm}$, in increments of $50.8 \mathrm{~mm}$. As depicted in Fig. 6, flow propagates from left to right. High pressure air is supplied on the upstream end, and a vacuum blower is located on the downstream end, such that the test window containing the acoustic liner is exposed to near-ambient pressure conditions for mean flows up to Mach 0.6. The surface of the test liner forms a portion $(609.6 \mathrm{~mm}$ for the current investigation) of the upper wall of the flow duct. Eighteen acoustic drivers are mounted upstream of the test window, and are used to generate tones (one frequency at a time) at up to $150 \mathrm{~dB}$ over a frequency range of 0.4 to $3.0 \mathrm{kHz}$.

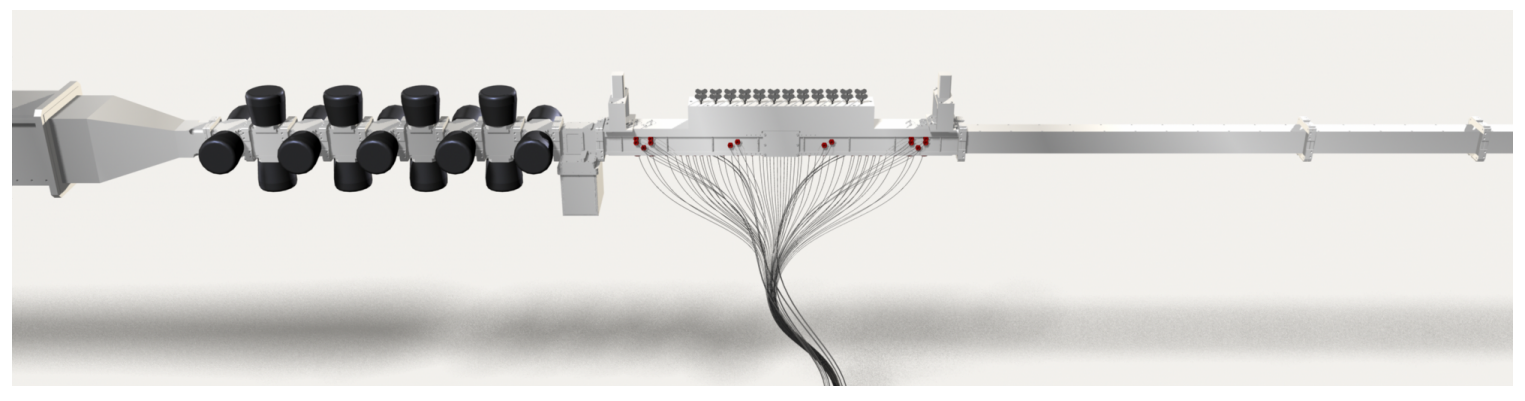

Figure 6: Sketch of Grazing Flow Impedance Tube.

Fifty-three microphones flush-mounted in the lower wall (opposite the liner) are used to measure the acoustic pressure field over the axial extent of $0 \leq x \leq L$, such that the technique described in Section II can be used to determine the impedance of the test liner. For each data acquisition, 2000 averages on each microphone channel (blocks of 2048 data points per average) are taken. To reduce the influence of flow noise, a cross-spectrum signal extraction method ${ }^{36}$ is used to determine the amplitudes and phases at each of the microphone locations relative to the amplitude and phase at the reference microphone location. For the current study, each liner configuration is tested using a tonal source (one frequency at a time) in the GFIT at centerline Mach numbers of 0.0 and 0.3 , with source sound pressure levels of 110 and $130 \mathrm{~dB}$, and at frequencies of 0.4 to $3.0 \mathrm{kHz}$ in $0.2 \mathrm{kHz}$ increments.

A dual-axis traverse probe system is used to measure the flow profile at selected axial planes in the GFIT. Two pitot probes are used for this measurement. One is shaped to allow detailed measurements close to the upper wall, and the other is shaped to allow corresponding measurements near the lower wall. The traverse system uses two stepping motors to successively move the pitot probe to preselected measurement locations, such that the full flow profile can be accurately determined. This system is used to acquire flow 
profiles upstream and downstream of the liner, from which average flow Mach numbers are computed. The upstream measurement plane is located $178 \mathrm{~mm}$ upstream of the computational domain source plane for acoustic computations, which is located at $x=0.0 \mathrm{~mm}$ (see Fig. 1). Similarly, the downstream measurement plane is located $178 \mathrm{~mm}$ downstream of the computational domain exit plane (at $x=L$ ). A linear fit between the two results is used to compute an average flow Mach number at the axial midpoint of the liner, and this average Mach number is used for the impedance eduction analysis. The traverse probe system is then removed for subsequent acoustic measurements.

\section{Results}

As mentioned earlier, successive tests in the NASA Langley raylometer, normal incidence tube (NIT) and grazing flow impedance tube (GFIT) are used for detailed evaluation of each liner configuration. Representative results of these tests are provided below. However, the complete database is available in electronic format upon request.

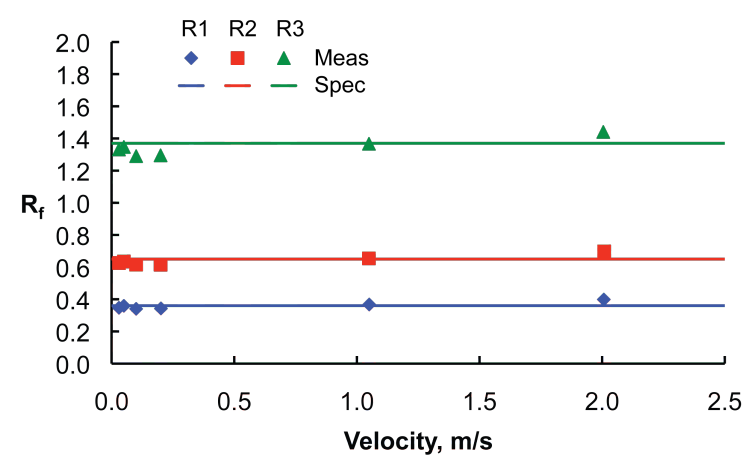

Figure 7: Comparison of measured and manufacturer's specified normalized, DC flow resistance as a function of mean flow velocity for R1, R2 and R3 wire mesh facesheets, respectively.

\section{A. DC Flow Resistance and Nonlinearity Factor}

Each wire mesh facesheet was tested in the raylometer to determine its DC flow resistance, $R_{f}$, and nonlinearity factor, $N L F$. Figure 7 provides normalized (relative to characteristic impedance of air, $\rho_{0} c_{0}$ ) $R_{f}$ as a function of velocity through the R1, R2 and R3 wire mesh sheets. For these tests, nominal flow velocities of $0.03,0.05,0.10,0.20,1.05$ and $2.00 \mathrm{~m} / \mathrm{s}$ were used. The lower velocities were included to gain insight into the response of the material at through-flow velocities of similar magnitude to typical acoustic particle velocities. Although a small amount of scatter is evident at the lowest velocities, the trends are generally as expected, i.e., minimal changes in DC flow resistance with increasing velocity.

The flow resistance measured at $1.05 \mathrm{~m} / \mathrm{s}$ is often used by manufacturers as the representative flow resistance of the material. The manufacturer's flow resistance was quoted as 150, 270 and 570 MKS Rayls $(0.36,0.65$ and $1.37 \rho c)$ for the R1, R2 and R3 wire mesh sheets, respectively. As shown in Fig. 7, the measured values almost identically match the manufacturer's specifications. Finally, the last value of interest is the nonlinearity factor, which is computed as

$$
N L F=\frac{R_{f}(2.0 \mathrm{~m} / \mathrm{s})}{R_{f}(0.2 \mathrm{~m} / \mathrm{s})}
$$

The NLF values of the R1, R2 and R3 wire mesh sheets are 1.16, 1.13 and 1.11 for R1, R2, and R3, respectively. These values are considerably lower than those measured with perforates used for conventional liner facesheets, which are typically greater than 2.0. This is a critical element of the methodology used to validate the results of the current investigation, as it indicates moderate changes in source SPL should 
result in minimal changes to the acoustic resistance spectra when these wire mesh sheets are used as liner facesheets.

\section{B. No-Flow Acoustic Impedance}

The four $50.8 \mathrm{~mm}$-deep samples described earlier were tested in the NASA NIT to determine their respective no-flow acoustic impedance spectra. The results measured for source SPLs of 110 and 130 dB are provided in Fig. 8. Except for the two lowest frequencies (0.4 and $0.6 \mathrm{kHz}$ ), the results are exactly as expected, i.e. the resistance is independent of frequency and equal to the DC flow resistance. At 0.4 and $0.6 \mathrm{kHz}$, the wavelengths are sufficiently long to increase the uncertainty in the results. The two microphones used in the Two-Microphone Method are $31.75 \mathrm{~mm}$ apart. Hence, as the wavelength increases (i.e., for lower frequencies), this microphone separation represents a reduced fraction of a wavelength. This increases the uncertainty of results at these frequencies.
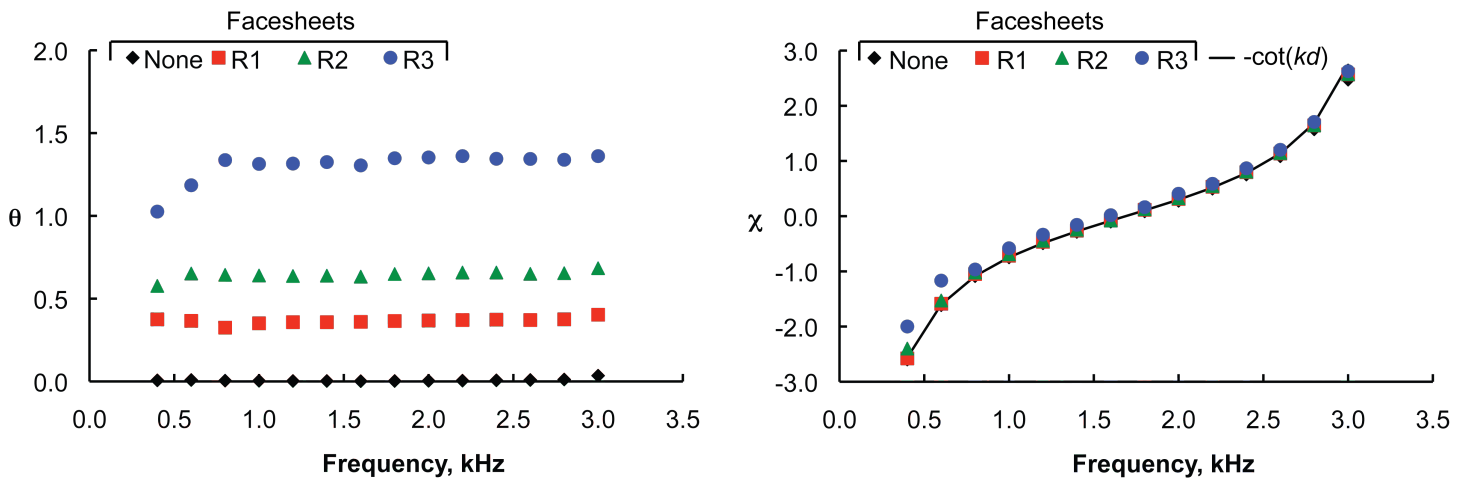

(a) Source SPL of $110 \mathrm{~dB}$
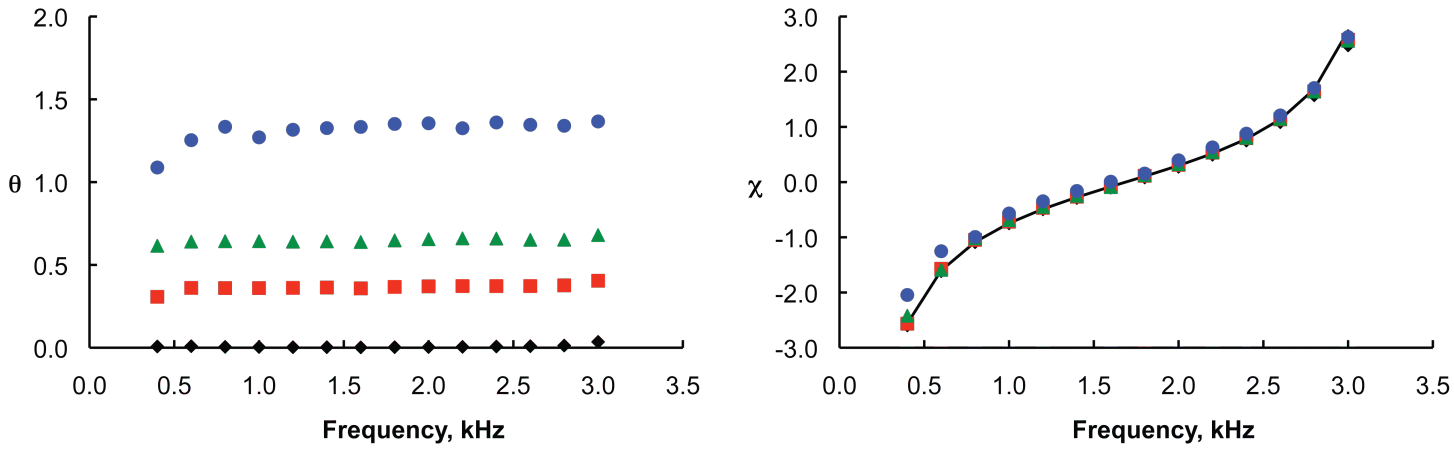

(b) Source SPL of $130 \mathrm{~dB}$

Figure 8: Effects of source SPL on no-flow acoustic impedances, $\zeta=\theta+i \chi$, for 50.8 mm-deep cavity with four configurations. Solid line represents computed cavity reactance.

The configuration with no facesheet (black diamonds) provides virtually no acoustic resistance, as the only contributions to acoustic resistance are from the scrubbing losses along the walls of the frame and the honeycomb in the cavity of the sample. This provides confidence that the acoustic resistances measured for the samples with facesheets are in fact due to the facesheets, i.e., effects of the frame and honeycomb walls on the acoustic resistance can be ignored. With the R1, R2 and R3 facesheets (red squares, green triangles and solid blue circles), the normalized resistances are $0.37,0.65$ and 1.33 , respectively. These normalized acoustic resistance values are nearly identical to the normalized DC flow resistance values for the three wire mesh facesheets, i.e., $0.36,0.65$ and 1.37 , respectively.

The normalized reactance spectra are nearly independent of the choice of facesheet. Except for the lowest two frequencies, all of the reactance values are nearly identical to the computed cavity reactance (solid line), i.e., $\chi(f)=-\cot (k d)$, where $k$ is the freespace wavenumber and $d$ is the cavity depth, respectively. This demonstrates that the wire mesh facesheets provide virtually no mass reactance, clearly different from that 

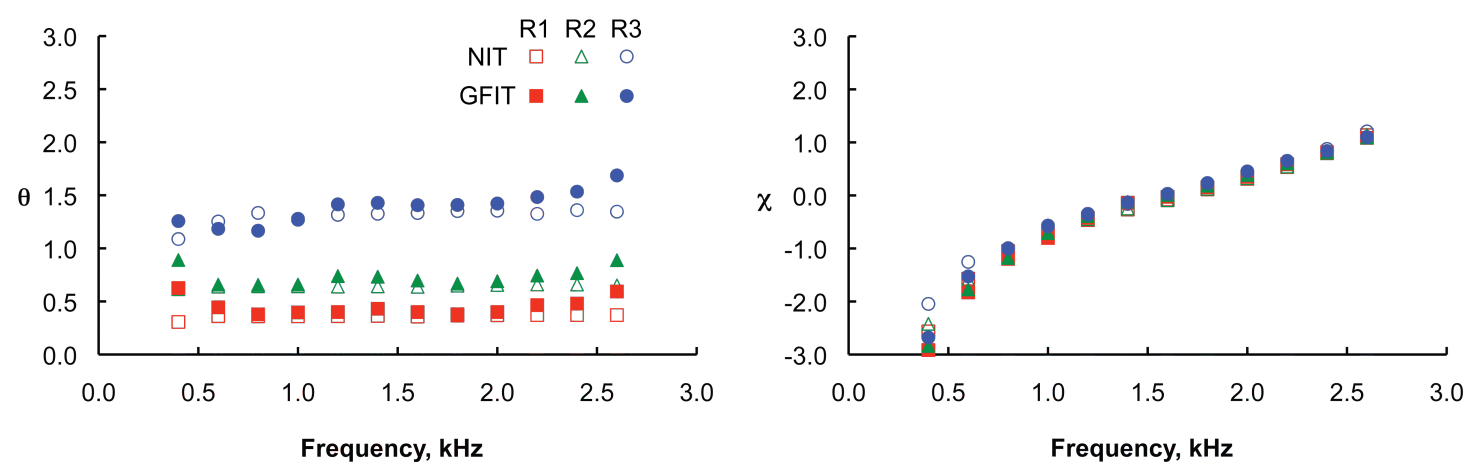

Figure 9: Comparison of impedances educed with the NIT and GFIT for $d=50.8 \mathrm{~mm}$ and $M_{0}=0.0$.

which would be expected for conventional perforate facesheets. Finally, the normalized impedance spectra are nearly identical for source SPLs of 110 and $130 \mathrm{~dB}$, which confirms that the wire mesh facesheets are linear with respect to source SPL within this regime. This result was expected, as the NLF values for the three wire mesh facesheets are all less than 1.2. This near-linearity result is valuable to the current study, as it indicates that (1) the effects of SPL on the acoustic impedance of a liner constructed with any of these facesheets should be minimal, and (2) coupling effects between the acoustic resistance and reactance components should be minimal for frequencies near resonance.

\section{Grazing Flow Acoustic Impedance}

The nine liner configurations (three wire mesh facesheets, each mounted onto three frames with different depths) described earlier were tested in the NASA grazing flow impedance tube (GFIT) at centerline Mach numbers of 0.0 and 0.3 , at $0.2 \mathrm{kHz}$ increments from 0.4 to $3.0 \mathrm{kHz}$, with source SPLs of 110 and $130 \mathrm{~dB}$. The impedance eduction technique described in section II was used to educe the normal incidence acoustic impedance spectra in the presence of grazing flow (designated as the grazing flow acoustic impedance). Figure 9 provides a comparison of impedance spectra educed with the NIT (open symbols) and GFIT (solid symbols) for frequencies from 0.4 to $2.6 \mathrm{kHz}$. The results are not presented for frequencies above $2.6 \mathrm{kHz}$ because the first higher-order mode is cut on for the no-flow condition. Tests were conducted with three samples of the same cavity depth $(d=50.8 \mathrm{~mm})$, at source SPLs of $130 \mathrm{~dB}$, with no flow $\left(M_{0}=0.0\right)$. The red squares, green triangles and blue circles provide results measured with the R1, R2 and R3 facesheets mounted onto the cavity, respectively. The results are observed to be nearly identical for the majority of the frequency range, thereby at least partially validating the impedance eduction process used with the GFIT.

Outlier acoustic resistances occur at 0.4 and $2.6 \mathrm{kHz}$. Fortunately, a review of the acoustic reactance spectrum offers insight into reasonable causes for these occurrences. For single-layer liners, such as are used in the current study, the reactance spectrum is dominated by the functional form of $-\cot (k d)$. For this configuration, resonance occurs at a frequency where the reactance experiences a positive-going zero crossing (for an $e^{i \omega t}$ time convention, as is used in this paper). For frequencies near a resonance, the liner is expected to provide increased attenuation. Conversely, anti-resonance occurs at a frequency where the reactance experiences a negative-going zero crossing. For single-layer liners, this means the reactance "jumps" from a large positive value (upper end of $-\cot (k d)$ curve) to a large negative value (lower end of the successive $-\cot (k d)$ curve) at anti-resonance. A review of Fig. 9 indicates that the frequency range of 0.4 to $2.6 \mathrm{kHz}$ does not include an anti-resonance, i.e., there are no "jumps" from large positive values to large negative values. However, it is apparent from the shape of the reactance spectrum that $0.4 \mathrm{kHz}$ is near the "beginning" of a $-\cot (k d)$ curve, and $2.6 \mathrm{kHz}$ is near the "end" of the same curve. Thus, both ends of the frequency range used in our test are near anti-resonances. This is important to our study, because the liner provides minimal attenuation for frequencies near anti-resonance. Specifically, the fact that 0.4 and $2.6 \mathrm{kHz}$ are near two antiresonances suggests that the attenuation will be minimal at these frequencies. Based on results observed in previous studies ${ }^{27,31}$ anomalous behavior is expected to occur for those configurations that produce minimal 
attenuation in the GFIT. This causes difficulty for any impedance eduction process, as there is a wide range of impedances that will result in minimal attenuation. Succesive results will demonstrate a method for overcoming this difficulty.
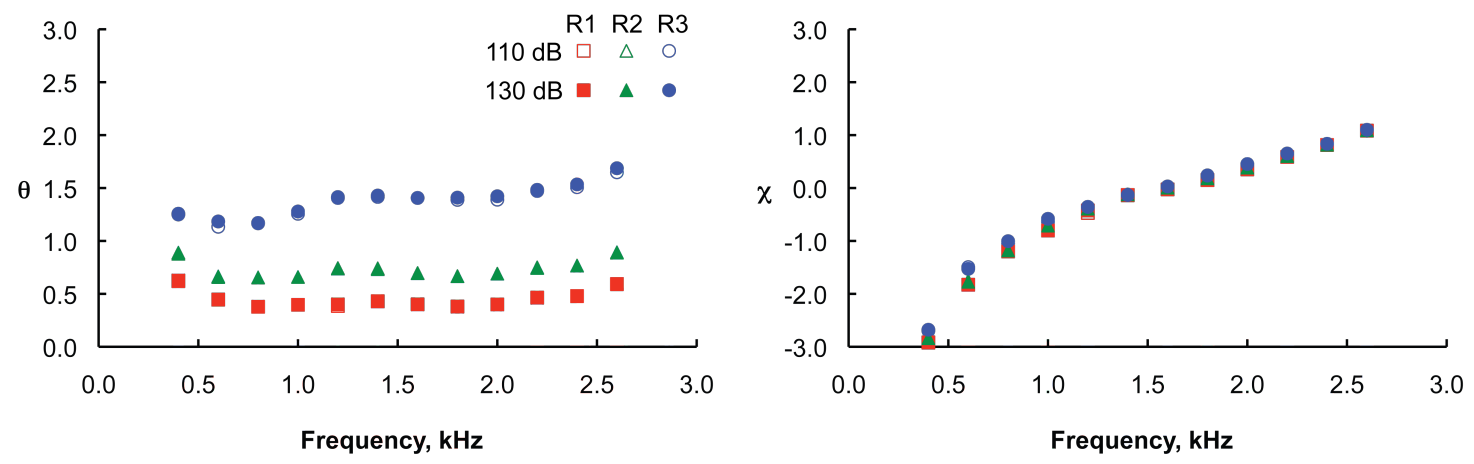

Figure 10: Effects of source SPL on impedances educed with the GFIT for $d=50.8 \mathrm{~mm}$ and $M_{0}=0.0$.
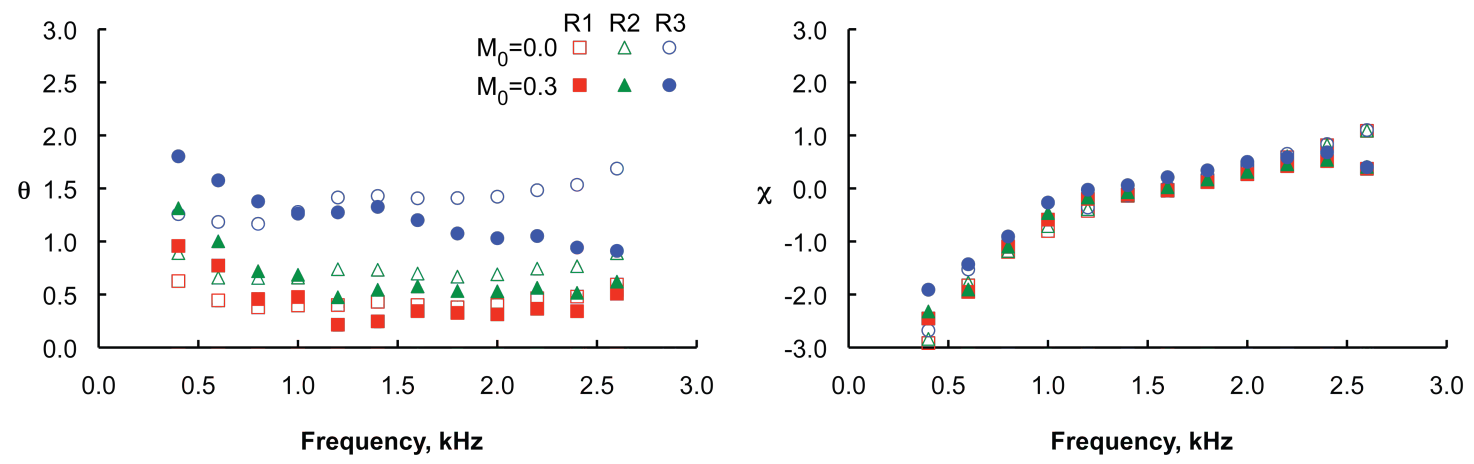

Figure 11: Effects of grazing flow Mach number on impedances educed with the GFIT for $d=50.8 \mathrm{~mm}$ and $S P L=130 \mathrm{~dB}$.

Figures 10 and 11 show the effects of source SPL and grazing flow Mach number on the impedance eduction process. Figure 10 provides a comparison of impedance spectra educed with the GFIT at source SPLs of 110 and $130 \mathrm{~dB}$. Each test was conducted with samples of the same cavity depth $(d=50.8 \mathrm{~mm})$, with no flow $\left(M_{0}=0.0\right)$. Again, the red squares, green triangles and blue circles provide results measured with the R1, R2 and R3 facesheets mounted onto the cavity, respectively. Clearly, the effects of source SPL are almost nonexistent, as the open symbols $(110 \mathrm{~dB})$ are almost completely obscured by the solid symbols $(130 \mathrm{~dB})$. This result is consistent with those acquired with the Raylometer for the three facesheets. Recall that nonlinearity factors for the three wire mesh facesheets were $1.16,1.13$ and 1.11 for R1, R2 and R3, respectively, indicating that moderate increases in the mean flow velocity through the facesheets resulted in minimal increases in DC flow resistance. Similarly, as the acoustic velocity through each facesheet is increased via an increase in source SPL, minimal increase in the acoustic resistance should be expected.

Figure 11 provides a comparison of impedance spectra educed at grazing flow centerline Mach numbers of 0.0 and 0.3 (average Mach number of 0.242 ). Results above $2.4 \mathrm{kHz}$ are not shown because the first higher-order mode cuts on below $2.6 \mathrm{kHz}$ for a centerline Mach number of 0.3 . There are two distinct frequency regimes of interest. The acoustic resistance increases with increasing Mach number for frequencies below resonance, and decreases with increasing Mach number above resonance. This is believed partially due to the assumption of uniform flow in the propagation code used for the impedance eduction process. As the DC flow resistance increases, this characteristic is more pronounced. For the samples with R1 and R2 
facesheets, the effects of Mach number on the educed acoustic resistance are small, albeit larger than the effects of source SPL, whereas the Mach number effects are much more signicant for the sample with the R3 facesheet. The corresponding effects of Mach number on the educed acoustic reactance are minimal.

Figure 12 shows the effects of cavity depth on the educed impedance spectra with the R3 facesheet sequentially mounted onto cavities with depths of 38.1, 50.8 and $76.2 \mathrm{~mm}$. Similar results were achieved with the R1 and R2 facesheets, but the R3 facesheet results are presented because this facesheet provides the highest acoustic resistance. A number of observations can be drawn from these results. First, note the existence of multiple values at $0.4 \mathrm{kHz}$. As mentioned earlier, four starting values were used for these impedance eductions to minimize issues associated with local minima. For a very limited number of test conditions, these different starting values resulted in different educed impedances, i.e., anomalous results. These different results are at least partially due to the minimal attenuation that occurs at this frequency, which is near an anti-resonance of the liner. By comparison of the numerical results for the four starting values, the "best" answer can generally be determined. For this case, only one of the four starting values provided an anomalous result. Thus, three of the values are overlaid in the figure, and the anomalous result (normalized resistance of 0.0 ) stands alone.

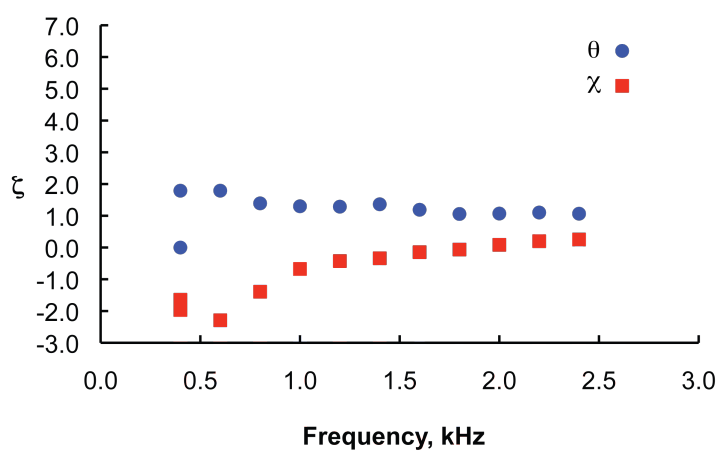

(a) Cavity depth, $d$, of $38.1 \mathrm{~mm}$.

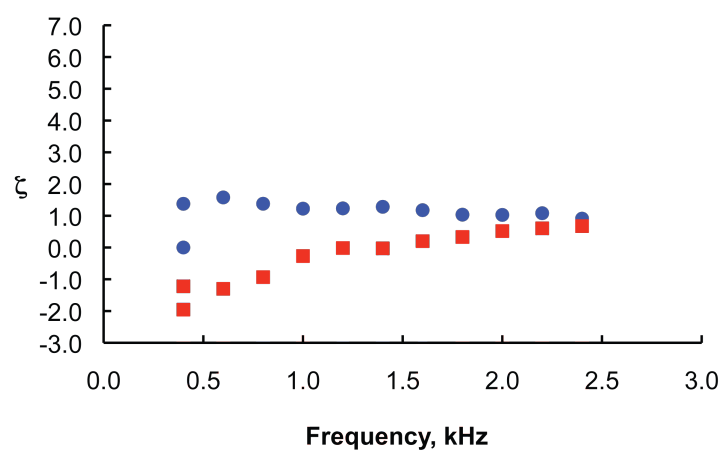

(b) Cavity depth, $d$, of $50.8 \mathrm{~mm}$.

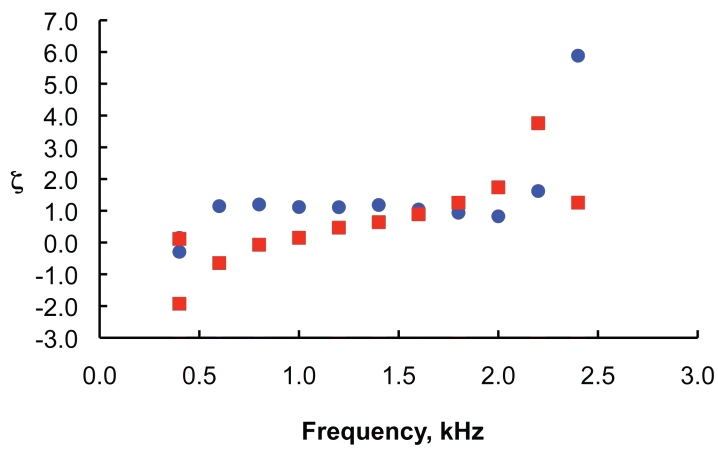

(c) Cavity depth, d, of $76.2 \mathrm{~mm}$.

Figure 12: Effects of cavity depth on impedances educed in the GFIT with R3 facesheet, $S P L=130 \mathrm{~dB}$, and $M_{0}=0.3$.

Generally speaking, the acoustic resistance is relatively independent of frequency, while the acoustic reactance follows a $-\cot (k d)$ pattern. Thus, impedance spectra that follow these patterns are assumed to be correct, while deviations from these patterns are generally considered to be anomalous. An example of this is shown for the $38.1 \mathrm{~mm}$ cavity depth in Fig. 12-a, where multiple reactances are educed at $0.4 \mathrm{kHz}$, and none of them follows the expected pattern. Clearly, these results are anomalous. Also, since both components of impedance (resistance and reactance) are educed simultaneously in the current methodology, the anomalous reactance value implies that the corresponding resistance value may also be anomalous. Thus, the "true" resistance at $0.4 \mathrm{kHz}$ should be determined with a different cavity depth. In particular, the $50.8 \mathrm{~mm}$ cavity depth results (Fig. 12-b) provide a better understanding at this frequency. Although there are multiple 
resistances and reactances at $0.4 \mathrm{kHz}$, the anomalous ones can be easily eliminated by visual inspection of the numerical results. In fact, this same result is determined by evaluating the results for each of the four starting values, indicating that the anomalous values are clearly outliers.

Another interesting feature is observed in Figure 12-c, in which the results presented are limited to those for which only plane waves propagate in the hardwall sections of the duct at a centerline Mach number of 0.3 . Note the significant increase in the acoustic resistance at 2.2 and $2.4 \mathrm{kHz}$, where the acoustic reactance approaches a large positive value, i.e., approaching an anti-resonance. As mentioned above, the acoustic liner provides minimal attenuation at frequencies near anti-resonance, and thus reduces the confidence of the educed impedance. Because of coupling between acoustic resistance and reactance, the acoustic resistance increases for frequencies near anti-resonance for all liners, and increases for frequencies near resonance ${ }^{37}$ for nonlinear liners. Hence, the increased resistance values at 2.2 and $2.4 \mathrm{kHz}$ are expected for this liner. This increased resistance is a function of the choice of liner depth, as opposed to an intrinsic property of the wire mesh facesheet. It should also be noted that while $0.4 \mathrm{kHz}$ is sufficiently close to anti-resonance to experience minimal attenuation, it is evidently sufficiently far from anti-resonance to avoid this coupling effect, such that the acoustic resistance at $0.4 \mathrm{kHz}$ is not increased.

Figure 13 provides a direct comparison of the acoustic resistance spectra educed with the R3 facesheet mounted onto each of the three cavities. It also shows the normalized DC flow resistance measured with the R3 facesheet in the raylometer (solid line). The anomalous dual values have been eliminated, as they are nonphysical (e.g., negative resistance). The resultant spectra can be combined to gain a better understanding of the acoustic resistance. As noted on multiple occasions, the fidelity of the educed impedance is reduced at frequencies where minimal attenuation is achieved. Thus, since $0.4 \mathrm{kHz}$ is near an anti-resonance for all three liners, this is the cause of variability of results for this test condition. For the liner with a cavity depth of $76.2 \mathrm{~mm}$, another anti-resonance occurs between 2.2 and $2.4 \mathrm{kHz}$, and the acoustic resistance is observed to increase significantly. As the attenuation is reduced at these frequencies, the fidelity of the educed impedance is reduced. As mentioned above, coupling of the acoustic resistance and reactance results in an increase of the acoustic resistance near anti-resonance. Hence, the increased resistance values at these frequencies are a function of the choice of liner depth, as opposed to an intrinsic property of the wire mesh facesheet.

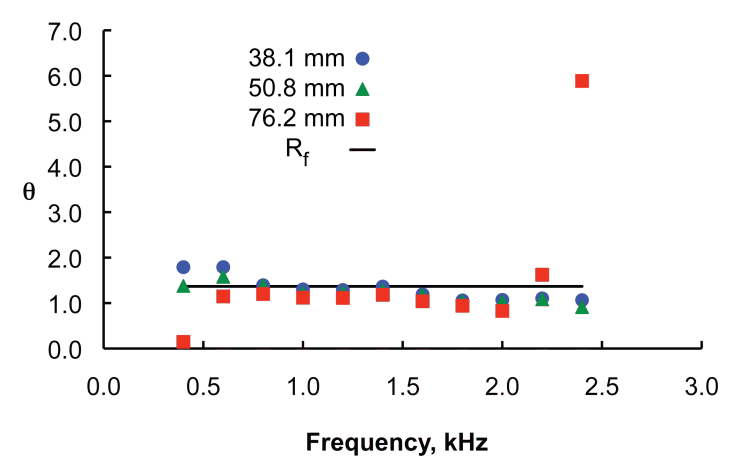

Figure 13: Effects of cavity depth on resistances educed with the GFIT for $S P L=130 \mathrm{~dB}, M_{0}=0.3$, and the R3 facesheet. Solid line represents normalized DC flow resistance of R3 facesheet.

Two items are observed to be of importance to the acoustic liner design process. First, the coupling of acoustic resistance and reactance is key to understanding impedance eduction results. The results of this study have demonstrated evidence of this coupling at frequencies near anti-resonance. For nonlinear liners, coupling also occurs at frequencies near resonance. However, this effect is significantly subdued for the nearlinear liners used in the current study. Second, a clear understanding of the final application (e.g., aircraft engine nacelle) for the acoustic liner is critical to determine how the impedance eduction data should be acquired and interpreted. If the cavity depth of the liner to be used in the final application is such that an anti-resonance occurs within the frequency range of interest, it is important to capture that effect in the flow duct test by testing a sample with that depth, such that the coupling effects are included in the evaluation. 
Although not demonstrated in the current study with nearly linear liners, this includes the need to capture resonance effects for nonlinear liners, e.g., conventional perforate-over-honeycomb, single-layer liners. On the other hand, there are many occasions when the goal is simply to educe the acoustic resistance of the facesheet without the effects of coupling between the acoustic resistance and reactance. Examples include:

1. The cavity depth for the final application is unknown, or is either too small or too large to be evaluated in the grazing flow impedance tube.

2. The facesheet is to be used with either a multi-layer liner or a single-layer, variable-depth liner, for which anti-resonance effects are significantly subdued.

3. The facesheet is to be used with an extended-reaction liner, for which the physics of the liner are much different.

4. The facesheet is to be used as a buried septum (interior layer) within a liner.

Impedances educed with multiple cavities can be combined to determine the facesheet acoustic resistance if anomalous results and coupling effects are correctly taken into account.

A comparison of the results provided in Figure 13 suggests the acoustic resistance of the R3 wire mesh facesheet can be successfully determined by combining the appropriate portions of the acoustic resistance spectra for the respective samples. Clearly, the results with the $76.2 \mathrm{~mm}$-deep cavity are dominated by acoustic resistance and reactance coupling effects at the frequency extremes, whereas the results educed with the $50.8 \mathrm{~mm}$-deep cavity appear suffiicient to describe the acoustic resistance provided by the R3 facesheet. Regardless, the additional data acquired with the other two cavity depths (38.1 and $76.2 \mathrm{~mm}$ ) provide increased confidence in the results. For linear liners, the appropriate portions consist of those frequency regimes away from anti-resonance. Similarly, it seems reasonable that frequencies near resonance would also need to be excluded due to coupling between the resistance and reactance for nonlinear liners. Confirmation of this hypothesis is expected to be the subject of a later investigation.

\section{Concluding Remarks}

It has long been understood that there is an optimum impedance for achieving maximum attenuation in a given waveguide geometry. Cremer used modal analysis to determine this optimum impedance in the absence of mean flow for a rectangular waveguide. Tester extended the model to include grazing flow effects, and also showed the attenuation to be a strong function of impedance for frequencies near this optimum, i.e., slight changes in liner impedance result in significant changes in attenuation. While uncertainty in the predicted attenuation increases with increasing attenuation, uncertainty in the educed impedance decreases. This study has demonstrated a way to exploit this knowledge to educe the liner impedance with increased confidence over a wide frequency range. Three sets of increasingly complex tests were conducted to study the impedance of three wire mesh sheets. Representative results of these tests were provided. However, the complete database is available in electronic format upon request.

Three wire mesh sheets were selected to cover a range of DC flow resistances. Raylometer measurements demonstrated the nonlinearity factor of each sheet to be less than 1.2, indicating they were very linear with respect to mean flow velocity. This suggests they should also be nearly linear with respect to acoustic velocity and, hence, the source sound pressure level. Each wire mesh sheet was then mounted combined with a frame and rigid backplate and tested in a normal incidence tube to determine its acoustic impedance. As expected, the acoustic reactance for each sample was properly described by $-\cot (k d)$, where $k$ is the freespace wavenumber, $d$ is the cavity depth, and an $e^{i \omega t}$ time convention is used. Except for frequencies near antiresonance, the acoustic resistance was demonstrated to be almost identical to the DC flow resistance. This excellent comparison included frequencies near resonance, thereby demonstrating the linearity of each liner. This linearity was also demonstrated via nearly identical impedance spectra measured with source sound pressure levels of 110 and $130 \mathrm{~dB}$.

The last set of tests was conducted with liners mounted in one wall of a grazing flow impedance tube (GFIT). Nine samples were tested in the GFIT, consisting of combinations of three wire mesh facesheets and three frames with varying depths. Each frame was filled with honeycomb core and terminated with a rigid backplate. Each sample was tested with two source sound pressure levels and no mean flow, and the 
NASA Langley impedance eduction method was used to educe the respective acoustic impedance spectra. For samples with identical frame thicknesses (cavity depths), the impedance spectra educed in the GFIT were well matched with those measured in the normal incidence tube, thereby demonstrating the efficacy of the impedance eduction method in the absence of mean flow. Tests were also conducted with grazing flow at a centerline Mach number of 0.3. Grazing flow was observed to increase the resistance for frequencies below resonance, and to decrease the resistance for frequencies above resonance. This effect was also observed to become more pronounced as the DC flow resistance of the facesheet was increased.

If the cavity depth of the liner to be used in the final application (e.g., engine nacelle) is known, it is preferable to conduct the flow impedance test with a sample of this depth, such that the effects of coupling between the acoustic resistance and reactance can be taken into account. However, if the the desire is to understand the facesheet such that it can be used in other configurations, it is best to conduct the test in a manner such that the effects of anti-resonances (and resonances for nonlinear liners) are subdued. For this latter case, the impedance spectra educed with different cavity depths can be combined to gain a better understanding of the acoustic resistance. For linear liners, as used in the current study, the educed resistance at frequencies near anti-resonances are suspect. These anti-resonances vary with cavity depth, such that each sample provides robust results over a different frequency range. Hence, a combination of results for multiple samples consisting of a single facesheet successively mounted onto frames with different depths can be used to determine the acoustic resistance of the facesheet. When combined with the acoustic reactance, observed to be weakly dependent on the source sound pressure level or the grazing flow Mach number, the acoustic impedance can be educed with confidence.

\section{Acknowledgements}

The authors would like to express appreciation to Carol Harrison, Jerry Lyle, Martha Brown and Brian Howerton for their assistance in the gathering of the data used in the current study. This work was funded by NASA's Subsonic Fixed Wing Project of the Fundamental Aeronautics Program.

\section{References}

${ }^{1}$ Envia, E. E., Huff, D. L., and Morrison, C. R., "Analytical assessment of stator sweep and lean in reducing rotor-stator tone noise," AIAA Paper 1996-1791, May 1996.

${ }^{2}$ Sutliff, D. L., Elliott, D. M., Jones, M. G., and Hartley, T. C., "Attenuation of FJ44 Turbofan Engine Noise with a Foam-Metal Liner Installed Over-the-Rotor," AIAA Paper 2009-3141, May 2009.

${ }^{3}$ Jones, M. G., Parrott, T. L., Sutliff, D. L., and Hughes, C. E., "Assessment of Soft Vane and Metal Foam Engine Noise Reduction Concepts," AIAA Paper 2009-3142, May 2009.

${ }^{4}$ Feder, E. and Dean, III, L. W., "Analytical and Experimental Studies for Predicting Noise Attenuation in Acoustically Treated Ducts for Turbofan Engines," NASA CR 1373, 1969.

${ }^{5}$ Syed, A. A., Yu, J., Kwan, H. W., and Chien, E., "The Steady Flow Resistance of Perforated Sheet Materials in High Speed Grazing Flows," NASA CR 2002-211749, 2002.

${ }^{6}$ Dean, P. D., "An In Situ Method Of Wall Acoustic Impedance Measurement In Flow Ducts," Journal of Sound and Vibration, Vol. 34, No. 1, 1974, pp. 97-130.

${ }^{7}$ Malmary, C., Carbonne, S., Auregan, Y., and Pagneux, V., "Acoustic Impedance Measurement With Grazing Flow," AIAA Paper 2001-2193, May 2001.

${ }^{8}$ Zandbergen, T., "On the Practical Use of Three-Microphone Technique for In-Situ Acoustic Impedance Measurements on Double Layer Flow Duct Liners," AIAA Paper 1981-2000, 1981.

${ }^{9}$ Lavieille, M., Simon, F., and Micheli, F., "Measurement of Liner Acoustic Impedance in a Shear Layer of a Subsonic Flow by Laser Doppler Velocimetry," Sapem 2005, December 2005.

${ }^{10}$ Betgen, B., Galland, M.-A., Piot, E., and Simon, F., "Characterization Of A Hybrid Active-Passive Liner By Means Of Laser Doppler Velocimetry," AIAA Paper 2010-3808, June 2010.

${ }^{11}$ Sawdy, D. T., Beckemeyer, R. J., and Patterson, J. D., "Analytical and Experimental Studies of an Optimum Multisegment Phased Liner Noise Suppression Concept," NASA CR 134960, 1976.

${ }^{12}$ Li, W., Ying, G., and Tongqing, W., "The 3-D Modal Method for Acoustic Impedance Determination in Rectangular Duct," AIAA Paper 2008-3015, May 2008.

${ }^{13}$ Elnady, T., Boden, H., and Elhadidi, B., "Validation of an Inverse Semi-Analytical Technique to Educe Liner Impedance," AIAA Journal, Vol. 47, No. 12, December 2009, pp. 2836-2844.

${ }^{14}$ Chung, J. Y. and Blaser, D. A., "Transfer function method of measuring in-duct acoustic properties: I. Theory," Journal of Acoustical Society of America, Vol. 68, 1980, pp. 907-921. 
${ }^{15}$ Jones, M. G. and Parrott, T. L., "Evaluation of a multi-point method for determining acoustic impedance," Journal of Mechanical Systems and Signal Processing, Vol. 3, No. 1, 1989, pp. 15-35.

${ }^{16}$ Lester, H. C. and Parrott, T. L., "Application of Finite Element Methodology for Computing Grazing Incidence Wave Structure in an Impedance Tube: Comparison with Experiment," AIAA Paper 79-0664, 1979.

${ }^{17}$ Elnady, T. and Boden, H., "On Semi-Empirical Liner Impedance Modeling With Grazing Flow," AIAA Paper 2003-3304, May 2003.

${ }^{18}$ Scofano, A., Murray, P. B., and Ferrante, P., "Back-Calculation of Liner Impedance Using Duct Insertion Loss Measurements and FEM Predictions," AIAA Paper 2007-3534, May 2007.

${ }^{19}$ Busse, S., Richter, C., Thiele, F. H., Heuwinkel, C., Enghardt, L., Rohle, I., Michel, U., Ferrante, P., and Scofano, A., "Impedance Deduction Based on Insertion Loss Measurements of Liners under Grazing Flow Conditions," AIAA Paper 2008-3014, May 2008.

${ }^{20}$ Fung, K.-Y., Jing, X., Lu, Z., and Yang, X., "Time-domain In Situ Characterization of Acoustic Liners in a Flow Duct," AIAA Journal, Vol. 47, No. 6, June 2009, pp. 1379-1387.

${ }^{21}$ Armstrong, D. L., Beckemeyer, R. J., and Olsen, R. F., "Impedance Measurements of Acoustic Duct Liners With Grazing Flow," Paper presented at 87th Meeting of the Acoustical Society of America 1999-1864, 1974.

${ }^{22}$ Succi, G., "A Method to Determine the Acoustical Properties of Locally and Nonlocally Reacting Duct Liners in Grazing Flow," NASA CR 165983, 1982.

${ }^{23}$ Parrott, T. L., Watson, W. R., and Jones, M. G., "Experimental Validation of a Two-Dimensional Shear-Flow Model for Determining Acoustic Impedance," NASA TP 2679, May 1987.

${ }^{24}$ Eversman, W. and Gallman, J. M., "Impedance Eduction with an Extended Search Procedure," , No. 2009-3235, May 2009.

${ }^{25}$ Watson, W. R., Tracy, M. B., Jones, M. G., and Parrott, T. L., "Impedance Eduction in the Presence of Shear Flow," AIAA Paper 2001-2263, May 2001.

${ }^{26}$ Jones, M. G., Watson, W. R., and Nark, D. M., "Effects of Flow Profile on Educed Acoustic Liner Impedance," AIAA Paper 2010-3763, June 2010.

${ }^{27}$ Jones, M. G., Watson, W. R., Tracy, M. B., and Parrott, T. L., "Comparison of Two Acoustic Waveguide Methods for Determining Liner Impedance," AIAA Paper 2001-2266, May 2001.

${ }^{28}$ Watson, W. R., Jones, M. G., and Parrott, T. L., "Validation of an Impedance Eduction Method in Flow," AIAA Journal, Vol. 37, No. 7, June 1998, pp. 818-824.

${ }^{29}$ Watson, W. R. and Jones, M. G., "Comparison of Convected Helmholtz and Euler Model for Impedance Eduction in Flow," AIAA Paper 2006-2643, May 2006.

${ }^{30}$ Watson, W. R., Jones, M. G., and Parrott, T. L., "Investigation of an Anomaly Observed in Impedance Eduction Techniques," AIAA Paper 2008-3013, May 2008

${ }^{31}$ Watson, W. R. and Jones, M. G., "Explanation of Anomalous Behavior Observed in Impedance Eduction Techniques Using Measured Data," AIAA Paper 2010-3766, May 2010.

${ }^{32}$ Cremer, L., "Theory Regarding the Attenuation of Sound Transmitted by Air in a Rectangular Duct with an Absorbing Wall, and the Maximum Attenuation Constant," Acustica, Vol. 3, 1953, pp. 249-263.

${ }^{33}$ Tester, B. J., "The Optimization of Sound Attenuation in Lined Ducts Containing Uniform, Axial, Subsonic, Mean Flow," Proceedings of the 7th International Congress on Acoustics, Budapest, Hungary, Vol. 4, (A73-1295 03-12), August 1971, pp. 209-212.

${ }^{34}$ Watson, W. R. and Jones, M. G., "Impedance Eduction in Ducts with Higher-Order Modes and Flow," AIAA Paper 2009-3236, May 2009.

${ }^{35}$ Myers, M. K., "On the Acoustic Boundary Condition in the Presence of Flow," Journal of Sound and Vibration, Vol. 71, No. 3, 1980, pp. 429-434.

${ }^{36}$ Bendat, J. S. and Piersol, A. G., Random Data: Analysis and Measurement Procedures, Wiley-Interscience, 1971.

${ }^{37}$ Motsinger, R. E. and Kraft, R. E., "Design and Performance of Duct Acoustic Treatment: Aeroacoustics of Flight Vehicles; Chapter 14, Vol. 2: Noise Control," NASA Reference Publication 1258, August 1991. 